\title{
Intervenção sobre Tabagismo Realizada por Cardiologista em Rotina Ambulatorial
}

\author{
Jaqueline Scholz Issa, Neusa Forti, Sergio D. Giannini, Jayme Diament
}

São Paulo, SP

\begin{abstract}
Objetivo - Avaliar a efetividade da intervenção sobre o tabagismo, realizada por médico cardiologista em rotina de ambulatório, utilizando a prescrição de adesivos de nicotina.

Métodos - Foram avaliados, consecutivamente, 100 pacientes (50 homens e 50 mulheres), incluindo consulta médica, aplicação de escore para definição do grau de dependência à nicotina, determinação da concentração de monóxido de carbono expirado e peso corpóreo. Os adesivos foram utilizados entre 8 e 12 semanas, com redução progressiva da concentração até a suspensão (concentrações de 21, 14 e 7mg)

Resultados - A taxa de abstinência um ano após o início do tratamento foi de $41 \%$, confirmada pela concentração do monóxido de carbono.

Conclusão - A intervenção sobre o tabagismo pode ser realizada em rotina de atendimento cardiológico com resultados satisfatórios. Os adesivos de nicotina são seguros, bem tolerados, e devem ser utilizados, mais freqüentemente, no auxílio aos fumantes, para deixarem de fumar.
\end{abstract}

Palavras-chaves: abordagem tabagismo, prevenção tabagismo

\section{Anti-Smoking Intervention Performed by Cardiologist During Ambulatory Care}

Purpose - The aim of this study was to evaluate the effectiveness of nicotine patches as a strategy to help patients quit smoking in the cardiovascular clinic.

Methods - The population studied was composed of 100 patients (50 women and 50 men). The strategy included medical consultation, Fangerstron escore application and prescription of nicotine patches. Nicotine patches were continuously used for 8 to 12 weeks, with progressive concentration reduction releasing 21,14 , and $7 m g /$ day.

Results - The abstinence rate one year later was $41 \%$ confirmed by carbon monoxide exhaled air concentration.

Conclusion - Nicotine patches are safe, and well tolerated and, for these reasons, should be more frenquently prescribed by cardiologists to help patients quit smoking.

Key-words: smoking cessation, smoking prevention

Arq Bras Cardiol, volume 70 (nº 4), 271-274, 1998

Considerando as taxas de morbi-mortalidade por doença cardiovascular, a interrupção do tabagismo é uma das principais estratégias para sua redução. A American Heart Association, em seu guia de Prevenção Primária de Doença Cardiovascular ${ }^{1}$, recomenda, entre as ações preventivas para redução da morbi-mortalidade por doença cardiovascular, a intervenção sobre o tabagismo com a prescrição de substitutos de nicotina.

A nicotina é a substância ativa responsável pela dependência dos indivíduos ao hábito de fumar ${ }^{2}$. Em 1992,

Instituto do Coração do Hospital das Clínicas - FMUSP

Correspondência: Jaqueline Scholz Issa - Incor - Ambulatório Divino Salvador Av. Divino Salvador, 308 - 04078-011 - São Paulo, SP

Recebido para publicação em 9/1/98

Aceito em 18/2/98 foram produzidos os adesivos transdérmicos de nicotina. Tornaram-se importante auxiliar no tratamento da dependência ao tabaco por reduzirem o aparecimento dos sintomas de abstinência. Ainda que estejam à disposição da classe médica, o uso tem sido restrito a pacientes sob orientação de grupos multiprofissionais e de clínicas especializadas.

No Brasil existe experiência relatada com atendimento multiprofissional a fumantes, envolvendo a participação de médico, nutricionista, psicóloga e enfermeira e a prescrição de nicotina transdérmica ${ }^{3}$. Não há trabalhos publicados que relatem experiência direta e isolada do médico com tratamentos específicos, objetivando a interrupção do tabagismo.

Esta investigação teve como objetivo principal verificar a viabilidade do uso do adesivo transdérmico em rotina de atendimento cardiológico, observando a frequiência de interrupção do tabagismo e o perfil de tolerância e segurança dos mesmos. 


\section{Métodos}

A casuística foi formada por 100 fumantes consecutivos que passaram por $1^{\mathrm{a}}$ consulta em ambulatório de rotina de atendimento cardiológico (Unidade Ambulatorial Divino Salvador do Incor). Destes, 50 eram homens (idade 43,9 $\pm 10,5$ ) anos e 50 mulheres (idade $42,4 \pm 10,6$ ) anos. Todos foram aconselhados a parar de fumar e convidados a participar de programa, utilizando os adesivos transdérmicos de nicotina. Apenas 10 indivíduos tinham coronariopatia, os demais eram portadores de hipertensão arterial , obesidade, dislipidemia, e/ou diabetes mellitus.

A rotina de atendimento compreendeu: avaliação clínica convencional; determinação do peso corpóreo $(\mathrm{kg})$, do grau de dependência à nicotina pelo escore de Fagerstrom ${ }^{4}$ (tab. I), da concentração do monóxido de carbono (CO) do ar expirado, por meio do equipamento mini-smokerlyzer (fig. 1). A determinação do CO foi realizada com objetivo de verificar a veracidade da informação sobre a taxa de abandono do fumo. Os indivíduos em abstinência devem apresentar concentração de CO inferior a 10 partes por milhão (ppm).

Os adesivos de nicotina possuem concentrações de $21,14 \mathrm{ou} 7 \mathrm{mg}$. Os pacientes iniciaram o tratamento usando $21 \mathrm{mg}$ se o escore fosse superior a $6 \mathrm{e} 14 \mathrm{mg}$ se inferior.

Os pacientes foram orientados para seguir algumas instruções: a) interrupção completa do tabagismo ao iniciar o uso dos adesivos; b) aplicação pela manhã, sempre na região superior do tórax, em local de menor concentração de pelos; c) substituição na manhã subseqüente; d) rodízio do local de aplicação.

O programa de prescrição dos adesivos compreendeu a utilização de concentrações progressivamente menores, utilizando cada uma ininterruptamente por 28 dias (quatro semanas). Assim, os que iniciaram com $21 \mathrm{mg}$, utilizaram essa dose por quatro semanas, a seguir passaram para a concentração de 14mg (quatro semanas) e depois para a de $7 \mathrm{mg}$ (quatro semanas).

As consultas foram realizadas a cada 21 dias no período de 12 ou de 8 semanas. Nelas, eram verificados: efeitos colaterais, o peso corpóreo, a concentração de monóxido de carbono e a adesão do paciente ao tratamento.

Os pacientes que concluíram a fase ativa de uso dos adesivos (8 ou 12 semanas) foram convocados para nova consulta um ano após o início do tratamento.
Segundo o comportamento ao longo da investigação, consideramos como: "desistentes", aqueles que concordaram a princípio com o tratamento, receberam os adesivos, mas efetivamente não iniciaram seu uso, alegando receio de não conseguir ficar sem fumar; "recaída", quando os pacientes voltaram a fumar após uso dos adesivos por pelo menos três dias; "sucesso", quando os pacientes concluíram a fase dos adesivos e, ao final de um ano, estavam sem fumar.

Foram utilizados os seguintes testes: 1) teste de associação para verificar eventual diferença na freqüência de adesão ao tratamento considerando as variáveis: idade, sexo, número de cigarros, concentração de monóxido de carbono nos diferentes grupos (desistência, recaída, sucesso);2) análise multivariada para verificar a influência das variáveis citadas. Considerou-se com significância estatísticap $<0,05$.

\section{Resultados}

Segundo o comportamento ao longo da investigação, foram identificados três grupos: grupo "desistentes"- 20 pacientes; grupo "recaída" - 39 pacientes; grupo "sucesso" - 41 pacientes. (tab. II).

A idade influenciou significativamente a taxa de sucesso de ambos os sexos $(p=0,02)$, indicando que quanto maior a faixa etária maior a probabilidade de sucesso (fig. 2).

A taxa de sucesso foi significativamente maior no sexo masculino que no feminino $(\mathrm{p}=0,008)$. A análise multivariada não evidenciou interferência do número de cigarros fumados $(\mathrm{p}=0,11)$ e da concentração de monóxido de carbono $(\mathrm{p}=0,8)$ nos diferentes grupos (tab. II).

Prurido leve no local da aplicação do adesivo foi referido por 20 pacientes. Esse sintoma foi tolerado com adoção de rodízio do local de aplicação.

Houve significativo ganho de peso tanto no sexo feminino $(3,9 \pm 3,4 \mathrm{~kg}, \mathrm{p}=0,009)$ quanto no masculino $(3,7 \pm 2,5 \mathrm{~kg}$, $\mathrm{p}=0,0001$ ) ao final de um ano do tratamento (tab. III).

Náusea foi referida por dois pacientes, após 20 dias do início do uso dos adesivos de $21 \mathrm{mg}$ e que melhorou após redução da dosagem para $14 \mathrm{mg}$.

Sonhos intensos e vivências foram referidos por 15 pacientes quando em uso de $21 \mathrm{mg}$. Somente em um paciente foi necessária a retirada noturna do adesivo. Nos demais não houve necessidade da interrupção noturna e nem mes-

\begin{tabular}{|c|c|c|c|}
\hline & 0 pontos & 1 ponto & 2 pontos \\
\hline 1.Quanto tempo após acordar você acende o primeiro cigarro? & depois de 30 minutos & em 30 minutos & \\
\hline 2. Você sente dificuldade em ficar sem fumar em locais proibidos? & Não & Sim & \\
\hline 3. Qual cigarro você se incomoda mais em não fumar? & qualquer um, exceto o primeiro & O primeiro do dia & \\
\hline 4. Quantos cigarros você fuma por dia? & $1-15$ & $16-25$ & 26 ou mais \\
\hline 5. Você fuma mais durante a manhã que no restante do dia? & Não & Sim & \\
\hline 6. Você fuma mesmo quando está doente? & Não & Sim & \\
\hline 7. Com que frequiência você traga o cigarro? & nunca & às vezes & sempre \\
\hline
\end{tabular}



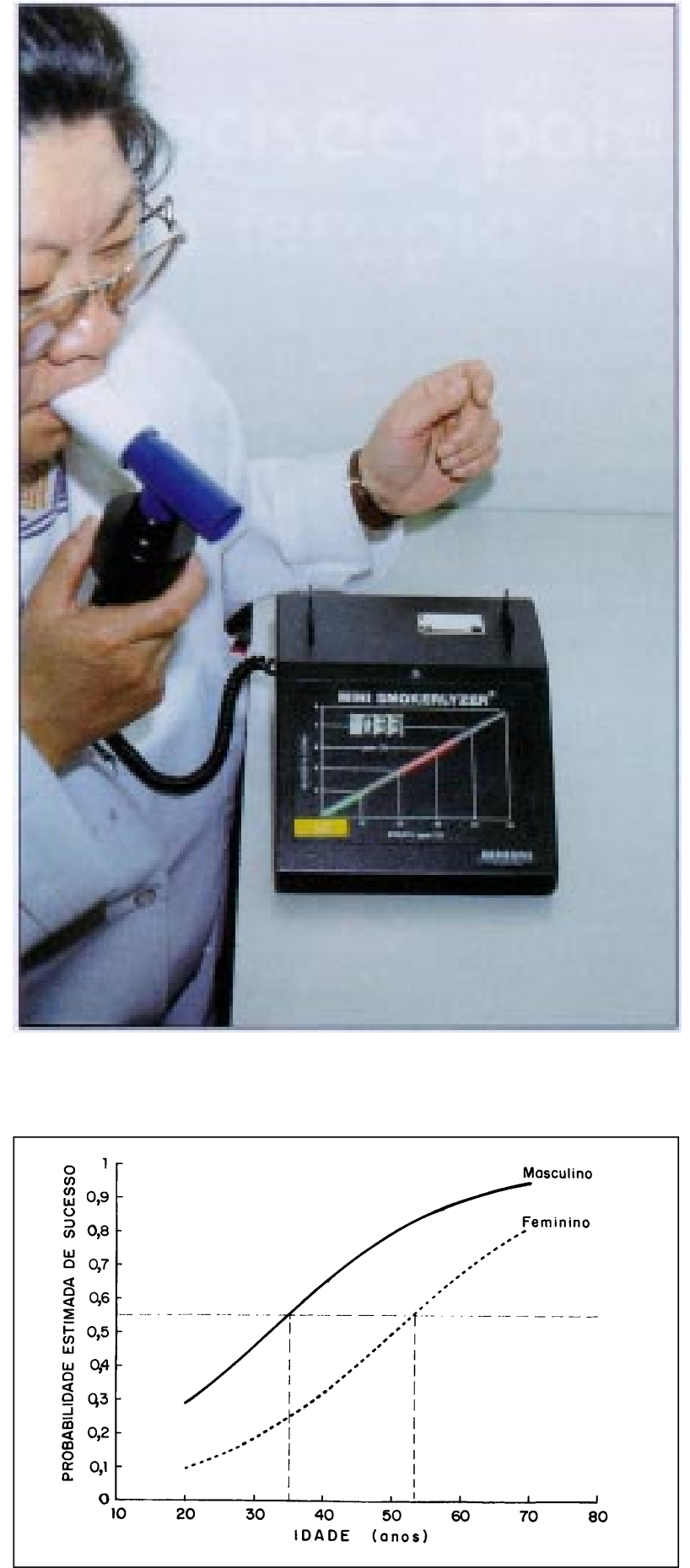

Fig 2-Probabilidade de sucesso da interrupção do tabagismo considerando idade e sexo.

mo a necessidade da redução precoce da dosagem, ocorrendo a regularização do sono após redução convencional da dosagem para $14 \mathrm{mg}$.

Não houve necessidade da interrupção da prescrição dos adesivos de nicotina em nenhum dos pacientes tratados.

\section{Discussão}

Algumas investigações mostram que $90 \%$ dos fumantes que interrompem o tabagismo o fazem por determinação pessoal, sem nenhuma forma de apoio, mas, também demonstram que de 100 indivíduos que se propõem a parar de fumar por conta própria, num intervalo de 10 anos, somente 10 têm sucesso permanente, com média de quatro a cinco tentativas prévias ${ }^{5}$. Considerando esses dados, fica evidente que, para otimizar resultados, há necessidade de se oferecer auxílio adicional aos fumantes. Especificamente, o uso de adesivos de nicotina pode desempenhar esse papel.

A nicotina é solúvel em água, rapidamente absorvida pelo trato respiratório, mucosa oral e pele. Como conseqüência, a concentração sérica aumenta rapidamente, existindo seletividade para pulmões, fígado, baço e cérebro, com deposição nesses órgãos. Da nicotina em circulação, 90 a $95 \%$ é metabolizada no fígado e apenas 5 a $10 \%$ é excretada pela via urinária. A ação farmacológica da nicotina no cérebro é complexa ${ }^{6}$, seu principal efeito é sobre o locus ceruleo. Esta região do sistema nervoso central é responsável pela regulação do binômio sono-vigília, pelas reações desencadeadas pelo estresse, e por manifestações do tipo psicossomático. Atua também no sistema dopa

minérgico mesolímbico ( região considerada como centro do prazer) determinando o comportamento compulsivo do fumante.

Os principais sinais e sintomas de abstinência à nicotina são ansiedade, sono inadequado, irritabilidade, impaciência, dificuldade de concentração, desejo incontrolável de fumar, raiva, distúrbios gastrintestinais, dor de cabeça e tonturas. Este conjunto de manifestações é conhecido e identificado como síndrome de abstinência à nicotina e catalogado no código internacional de doenças (CID 10) e no Diagnostic and Statistical Manual of Mental Disorders da Associação Americana de Psiquiatria 1987, (DSM R III 1987) ${ }^{7}$

Os estudos comparativos utilizando placebo e adesivos transdérmicos demostraram sua maior eficácia. A taxa de abandono obtida com placebo varia de 5 a $10 \%$ e com adesivos de 18 a $77 \%{ }^{8}$. Fiore e col $^{9}$ confirmaram seus achados realizando metanálise de vários estudos publicados. A variação na taxa de abandono com os adesivos é determinada principalmente por seleção de pacientes com diferentes graus de motivação para o tratamento.

A taxa de abandono nesse estudo foi de $41 \%$ e, quando a comparamos com a obtida por grupo multiprofissional $(42 \%)^{3}$, concluímos que nosso resultado é muito satisfatório.

Constatamos boa tolerância e segurança com o uso dos adesivos. Apesar da nossa casuística ter sido composta somente por 10 coronariopatas, em nenhum houve agravamento do quadro anginoso. Estudos indicam baixo risco de complicações com uso dos adesivos em cardiopatas, sendo demostrada expressiva redução da isquemia miocárdica esforço induzida (avaliada pelo do teste com tálio 201 SPECT) em coronariopatas com seu uso ${ }^{10}$.

É constante a observação de que os fumantes têm em média peso corpóreo menor que não fumantes e que a interrupção do tabagismo determina expressivo ganho do peso. Justifica-se este comportamento por algumas das ações da nicotina, dentre as quais: capacidade de determinar sacie- 


\begin{tabular}{|c|c|c|c|c|c|}
\hline \multirow{3}{*}{ Grupos } & \multicolumn{4}{|c|}{ Tabela II - Características dos grupos } & \multirow{3}{*}{$\begin{array}{c}\text { Monóxido } \\
\text { de carbono (ppm) }\end{array}$} \\
\hline & Idade (anos) & \multicolumn{2}{|c|}{ Sexo } & \multirow{2}{*}{$\begin{array}{l}\text { Consumo } \\
\text { cigarros/dia }\end{array}$} & \\
\hline & & Feminino & Masculino & & \\
\hline Desistência & $43,4 \pm 13,9$ & 7 & 13 & $28,2 \pm 15,7$ & $30,1 \pm 13,1$ \\
\hline Recaída & $39,7 \pm 8,5$ & 27 & 12 & $23,6 \pm 9,4$ & $27,4 \pm 9,9$ \\
\hline Sucesso & $46,3 \pm 11,2$ & 16 & 25 & $22,4 \pm 7,4$ & $27,8 \pm 12,7$ \\
\hline
\end{tabular}

\begin{tabular}{|lcc|}
\hline \multicolumn{2}{|c|}{ Tabela III - Peso corpóreo antes e após interrupção do tabagismo } \\
\hline & $\begin{array}{c}\text { Peso Inicial } \\
(\mathrm{kg}) \text { média } \pm \mathrm{dp}\end{array}$ & $\begin{array}{c}\text { Peso após 1 ano } \\
(\mathrm{kg}) \text { média } \pm \mathrm{dp}\end{array}$ \\
\hline Sexo feminino & $63,6 \pm 11,1$ & $67,5 \pm 9,6$ \\
Sexo masculino & $77,1 \pm 15,7$ & $80,8 \pm 16,7$ \\
\hline
\end{tabular}

dade; capacidade de diminuir o paladar e o olfato; efeito termogênico. Além das referidas ações, ao haver interrupção do tabagismo, há imediata substituição do cigarro por alimentos ${ }^{11}$. Estudo conduzido pelo National Center for Health Statistics (NCHS - USA) com 5247 adultos $>35$ anos de 1988 a 1991 constatou que o ganho de peso em 10 anos foi 4,4kg em homens ex-fumantes e 2,4kg em não fumantes e, entre as mulheres, $5 \mathrm{~kg}$ nas ex-fumantes e $2 \mathrm{~kg}$ nas não fumantes ${ }^{12}$.

O ganho de peso corpóreo nos programas de interrupção do tabagismo implica na necessidade dos médicos orientarem os pacientes a incrementar a atividade física e terem precaução de adotar dietas hipocalóricas para minimizar esse efeito ${ }^{13}$.

Possivelmente, o ganho de peso justifique, em parte, a maior taxa de recaídas entre as mulheres observada neste estudo. Embora tenha ocorrido ganho de peso em ambos os sexos, sabemos que entre as mulheres essa preocupação é evidentemente maior, talvez justificando a maior taxa de sucesso entre os homens observada também em outras publicações ${ }^{14}$.

A diferença de obtenção de sucesso em faixas etárias mais elevadas, observada em nosso estudo desmistifica conceito popular que quanto mais tempo de fumo maior a dificuldade para parar de fumar, e reforça a importância do aspecto motivador na determinação do sucesso. Possivelmente, indivíduos mais velhos tenham maior preocupação com doença que os mais jovens, o que poderia determinar maior motivação para a interrupção do tabagismo.

Diante do exposto, pode-se concluir que os cardiologistas dispõem de recurso seguro na abordagem do tabagismo, podendo obter resultados satisfatórios quando se propuserem a intervir sobre ele, propiciando redução na morbi-mortalidade por doença cardiovascular e limitando o encaminhamento de fumantes resistentes para clínicas especializadas.

\section{Agradecimentos}

Ao Laboratório Novartis Biociências S/A pela doação dos adesivos de nicotina utilizados neste estudo.

\section{Referências}

1. Grundy SM, Balady GJ, Criqui MH et al - Guide to Prevention of Cardiovascular Diseases. A Statemant for Healthcare Professionals from the Task Force on Risk Reduction. Circulation 1997; 95: 2329-31.

2. US Department of Health and Human Services - The health consequences of smoking : nicotine addiction. A Report of Surgeon General. Public Health Service, Centers for Disease Control, Center for Health Promotion and Education, Office on Smoking and Health, USDHHS publ. no (CDC) 1988; 88-8406:523.

3. Labbadia EM, Ismael SC, Fernandes ND et al - Atendimento multiprofissional ao tabagista: uma opção terapêutica. Rev Soc Cardiol ESP 1995; 5(supl A): 6-11.

4. Fagerstrom KO-Measuring degree of physical dependence on tobacco smoking with reference to individualization of treatment. Addictive Beh 1978; 3: 235-41.

5. Fiore MC, Novotny TE, Pierce JP et al - Methods used to quit smoking in the United States: do cessation programs help? JAMA 1990; 263: 2760-65.

6. Grenhoff J, Svensson TH-Pharmacology of nicotine. Br J Addict 1989: 84; 47792.

7. American Psychiatric Association - Diagnostic and Statistical Manual of Mental Disorders (revised), $3^{\text {rd }}$ ed (DSM III R). Washington, DC: American Psychiatric Association, 1987.
8. Fiore MC, Jorenby DE, Baker TB et al - Tobacco dependence and the nicotine patch. Clinical guidelines for effective use. JAMA 1992; 268: 2687-94.

9. Fiore MC, Smith SS, Jorenby DE et al - The effectiveness of the nicotine patch for smoking cessation: meta -analysis. JAMA 1994; 271: 1940-7.

10. Mahmarian JJ, Moyé LA, Nasser GA et al - Nicotine patch therapy in smoking cessation reduces the extent of exercise-induces myocardial ischemia. J Am Coll Cardiol 1997; 30: 125-30

11. Sachs DPL, Leischow SJ - Pharmacologic Approaches to Smoking Cessation. Clinics in Chest Medicine 1991; 12: 769-91.

12. Flegal KM, Troiano RP, Pamuk ER et al - The influence of smoking cessation on the prevalence of overweight in the United States. N Engl J Med 1995; 333: 1165-70.

13. Kawachi I, Troisi RJ, Rotnizky AG et al - Can physical activity minimize weight gain in women after smoking cessation? Am J Public Health 1996; 86: 9991004.

14. Gritz ER, Nielsen IR, Brooks LA - Smoking cessation and gender: the influence of physiological, psychological, and behavioral factors. J Am Med Women Assoc 1996; 51: 35-42. 